\title{
CYCLIC COHOMOLOGY AFTER THE EXCISION THEOREM OF CUNTZ AND QUILLEN
}

\author{
JACEK BRODZKI
}

\section{INTRODUCTION}

This article surveys developments in cyclic cohomology that were made possible by the Cuntz-Quillen theorem on excision in periodic cyclic homology and cohomology.

Periodic cyclic cohomology was introduced by Connes in his ground-breaking article [3]. It is Morita and homotopy invariant, two properties which it has in common with topological K-theory. Moreover, it admits a pairing with K-theory similar to that provided by the Chern character. A very important property of topological K-theory is excision, that is the existence of an exact sequence of length six associated with an extension of $\mathrm{C}^{*}$-algebras. These three properties, together with a normalisation condition, essentially characterise K-theory and are used in essentially all explicit calculations. It was not known for a long time if an excision theorem holds in periodic cyclic cohomology. An important result in this direction was established by Wodzicki [32], who proved that excision holds for an algebra extension $\mathrm{O} \rightarrow \mathrm{S} \rightarrow \mathrm{P} \rightarrow \mathrm{Q} \rightarrow \mathrm{O}$ in which the ideal $\mathrm{S}$ is $\mathrm{H}$-unital (homologically unital). While this was a very important breakthrough, many natural extensions do not have this property. For example, in the universal extension introduced by Cuntz in [5]

$$
0 \rightarrow \mathrm{IA} \rightarrow \mathrm{QA} \rightarrow \mathrm{A} \rightarrow 0
$$

the ideal $I A$ is not $H$-unital in general. Here the universal algebra $Q A=A * A$ is the free product of the algebra $A$ by itself, and the map $Q A \rightarrow A$ is the 'folding map' which identifies the two copies of $A$. In a remarkable paper [9], Cuntz and Quillen proved the excision property for their bivariant periodic cyclic homology $\mathrm{HP}_{*}(A, B)$ and for all algebra extensions. As in other bivariant theories, the connecting homomorphisms in the six-term exact sequence are given by the composition product with a class in $\mathrm{HP}_{1}$, which can be regarded as the fundamental class of the extension.

One immediate corollary of the excision theorem is the existence of a bivariant Chern character from an algebraic version of KK-theory to bivariant periodic cyclic homology which is compatible with the composition product. A very important example of the power of this result was provided by Nistor [23] who used it to provide a new proof of the ConnesMoscovici index theorem for coverings.

The importance of the excision result in the purely algebraic case cannot be overestimated. Moreover, the approach taken by Cuntz and Quillen relies on their new formalism which provided a unified description of all cyclic-type homology theories and proved to be a perfect framework to study extensions of these theories to other classes of algebras, which is the focus of this article. For example, the algebra $C^{\infty}(M)$ of smooth functions on a compact manifold has a natural Fréchet topology, which needs to be incorporated into the theory.

Another important conceptual problem was posed by Connes' entire cyclic cohomology. It was clear from the first construction of periodic cyclic cohomology by Connes in [3] that

2010 Mathematics Subject Classification. Primary 19D55, Secondary 48L80, 58B34, 46A17. ter.

Keywords: Bivariant cyclic theories, excision, Cuntz-Quillen theorem, bornological algebras, Chern charac-

Partially supported by an EPSRC grant EP/I016945/1. 
one should expect the existence of infinite dimensional cocycles. From this point of view, the periodic cyclic cohomology would be suitable for finite dimensional spaces, while this new cohomology theory would be able to deal with infinite dimensional ones. The entire cyclic cohomology of a Banach algebra $A$ is constructed using infinite $\mathbb{Z} / 2 \mathbb{Z}$-graded inhomogeneous cocycles $\left(\phi_{2 n}\right)_{n \in \mathbb{N}}$, where each $\phi_{2 n}$ is a $(2 n+1)$-linear functional on the algebra $A$ and which together satisfy the condition that the radius of convergence of the series

$$
\sum_{n} \frac{\left\|\phi_{2 n}\right\|}{n !} z^{n}
$$

is infinite. Here we have to deal with two pieces of data: the topology on the algebra $A$ and the growth condition imposed on cocycles. Meyer's surprising use of bornology allowed him to create a new type of cyclic theory, the bivariant analytic cyclic cohomology, where a main datum is a choice of bornology on the algebra rather than a topology. The entire cyclic cohomology fits very naturally into this context. Bornology, which had been part of functional analysis for a long time, was never used in the context of cohomology of topological algebras before Meyer's work, and most definitely not in any systematic way.

In another development, Puschnigg's local cyclic cohomology can be defined for Banach and $C^{*}$-algebras and possesses very useful density and continuity properties. We will discuss both theories in some detail below. Let us also mention that Voigt developed an equivariant version of the local theory [30][31].

In what follows we shall sketch a few results that illustrate the progress that was made possible by the Cuntz-Quillen excision theorem and its natural extensions to other contexts. We have chosen problems that illustrate both the power of the theory and how it might be applied to provide interesting insights into problems in noncommutative geometry, more broadly understood. The present exposition is of course incomplete, as the work that resulted from this important theorem is impossible to describe in a short article.

I was privileged to witness, as Daniel Quillen's DPhil student at Oxford, the long and difficult development of the formalism of cyclic cohomology, based on non commutative differential forms, which was later a key ingredient in the Cuntz-Quillen excision theorem. In his work Dan was driven by a deep desire to arrive at the clearest possible understanding of a mathematical problem. This is probably best described in his own words, written in the introduction to his paper [28], dedicated to Grothendieck:

[Grothendieck's] work has been of immense importance in my own development as a mathematician, because the simple powerful concepts and formalisms he found to understand one area of mathematics have often turned out to be very fruitful in other contexts. It is clear from the present paper, with its extensive use of explicit complexes and formulas, that a true Grothendieck understanding of cyclic cohomology remains a goal for the future.

This was the motivation driving the development of the Cuntz-Quillen formalism for cyclic type cohomology theories and I believe that the theory is now very close to attaining this high ideal.

Daniel Quillen was a great mathematician. He was also a wonderful person, who was warm, humble, and generous with his time and his ideas. It was a great honour for me to be able to spend a few years in his company.

\section{THE X-COMPLEX}

A very important legacy of the work of Cuntz and Quillen is that all cyclic type homology theories of an algebra $A$ can be defined using a $\mathbb{Z} / 2 \mathbb{Z}$-graded complex associated with $A$. This complex, loosely speaking, is constructed using a certain deformation of the tensor algebra of $A$. We begin by describing the purely algebraic case; this is covered in detail in the article by Cuntz in this volume [11] to which we refer for further information. 
Let $A$ be an algebra, with or without unit. First recall that the differential graded algebra $\Omega A$ of noncommutative differential forms associated with $A$ is generated by the elements of $A$ together with symbols $d a$ for each $a$ in $A$, such that $d a$ is linear in a and satisfies the Leibniz rule $d(a b)=(d a) b+a d b$. In degree $n \geq 1, \Omega^{n} A=\widetilde{A} \otimes A^{\otimes n}$, where $\widetilde{A}$ is the algebra $A$ with a unit adjoined. Thus, in degree $n \geq 1, \Omega^{n} A=A^{\otimes n+1} \oplus A^{\otimes n}$, and so elements of $\Omega^{n} A$ are linear combinations of differential forms $a_{0} d a_{1} \ldots d a_{n}$ and $d a_{1} \ldots d a_{n}$, with $a_{i}$ in $A$. In degree zero we put $\Omega^{0} A=A$. To define the structure of a differential complex on the graded space $\Omega A$ we introduce two operators

$$
b=\left(\begin{array}{cc}
b & 1-\lambda \\
0 & -b^{\prime}
\end{array}\right), \quad B=\left(\begin{array}{cc}
0 & 0 \\
N & 0
\end{array}\right)
$$

where the operators $b^{\prime}, b, \lambda, N$ have their usual meaning, cf. [7, p.9].

Let us denote by $\widehat{\Omega} A$ the infinite direct product $\widehat{\Omega} A=\prod_{n} \Omega^{n} A$. This can be regarded as the completion of $\Omega A$ in the natural adic topology generated by powers of the ideal IA consisting of the differential forms of degree at least one. The differentials $b$ and $B$ extend to operators on $\widehat{\Omega} A$.

Now for any two algebras $A$ and $B$ one defines bivariant cyclic homology by

$$
\mathrm{HP}_{*}(A, B)=\mathrm{H}_{*}(\operatorname{Hom}(\widehat{\Omega} A, \widehat{\Omega} B)) .
$$

Here Hom denotes the complex of continuous homomorphisms between the two complexes of differential forms.

While this definition is natural, the $X$ complex of Cuntz and Quillen has better formal properties. The $X$-complex $X(A)$ associated with an algebra $A$ is the $\mathbb{Z} / 2 \mathbb{Z}$-graded complex

$$
A \underset{\mathrm{b}}{\stackrel{\natural \mathrm{d}}{\rightleftarrows}} \Omega^{1} A_{\natural}
$$

where $\Omega^{1} A_{\natural}=\Omega^{1} A /\left[A, \Omega^{1} A\right]=\Omega^{1} A / b\left(\Omega^{2} A\right)$, and $\natural: \Omega^{1} A \rightarrow \Omega^{1} A_{\natural}$ is the canonical projection map, compare [7, p. 21] and Cuntz's article in the present volume.

In order to obtain an interesting homology theory we need to apply the above construction to the non-unital tensor algebra TA of the algebra A. While it seems at first sight that this will lead to a huge and unwieldy object, it turns out in fact, thanks to a key result of Cuntz and Quillen [10, Theorem 5.5], that the resulting complex is a deformation of the mixed complex $(\Omega A, b, B)$. This observation rests on the fact that the tensor algebra of any algebra $A$ can be identified with the even part of the algebra $\Omega A$ equipped with the Fedosov product

$$
\omega \circ \eta=\omega \eta-(-1)^{\operatorname{deg} \omega} \mathrm{d} \omega \mathrm{d} \eta
$$

for any $\omega, \eta \in \Omega A$.

Proposition 2.1. ([10][7, Thm. 2.29, p. 24]) For any algebra A the X-complex of the tensor algebra TA of A is isomorphic to a complex of the form

$$
\Omega^{e v} \mathrm{~A} \underset{\beta}{\stackrel{\delta}{\rightleftarrows}} \Omega^{\text {odd }} \mathrm{A}
$$

where the differentials $\beta$ and $\delta$ can be explicitly determined in terms of the differentials $\mathrm{b}$ and $\mathrm{B}$.

It follows from this result that the periodic cyclic homology introduced above can be computed as the homology of a bivariant differential complex arising from the $\mathrm{X}$-complex:

$$
\mathrm{HP}_{*}(\mathrm{~A}, \mathrm{~B})=\mathrm{H}_{*}(\operatorname{Hom}(\mathrm{X}(\mathrm{TA}), \mathrm{X}(\mathrm{TB})) .
$$

An important advantage of this construction is that it allows extensions to other contexts, as we shall see in what follows. The Cuntz-Quillen excision theorem can now be stated as follows. 
Theorem 2.2. Let $\mathrm{O} \rightarrow \mathrm{S} \rightarrow \mathrm{P} \rightarrow \mathrm{Q} \rightarrow 0$ be an extension of algebras, and let $\mathrm{A}$ be an algebra. There are two natural six-term exact sequences

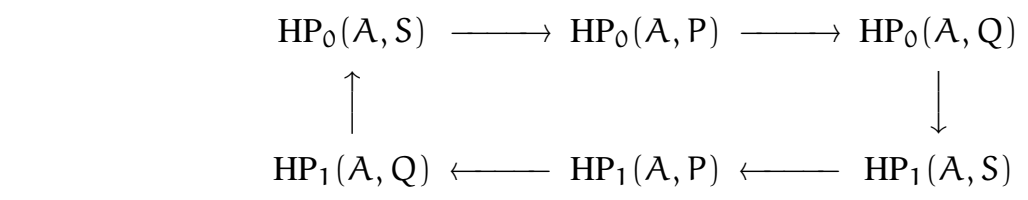

and

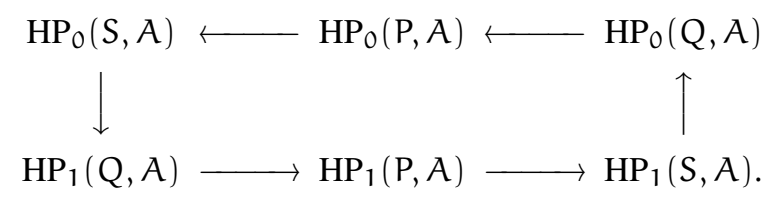

The horizontal maps in this diagram are induced by the maps in the extension. We shall outline a construction of the vertical arrows below.

Let us remark here that Nistor showed [24] that the Chern character $c h: K_{i}^{a l g}(A) \rightarrow$ $\mathrm{HP}_{i}(A)$, for $i=0,1$, mentioned in the introduction, provides a natural transformation from the six-term exact sequence in lower algebraic K-theory to the six term exact sequence in periodic cyclic homology. (This is the first of the two sequences above taken with $A=\mathbb{C}$.). Moreover, he proved that the boundary map $\mathrm{HP}_{1}(\mathrm{Q}) \rightarrow \mathrm{HP}_{0}(\mathrm{~S})$ is compatible, via the Chern character, with the connecting homomorphism Ind : $K_{1}^{\mathrm{alg}}(\mathrm{Q}) \rightarrow \mathrm{K}_{0}(\mathrm{~S})$, and provided an interpretation of this in terms of a higher index theorem.

\section{TOPOLOGICAL AND BORNOLOGICAL ALGEBRAS}

Cyclic type homology groups of an algebra $A$ are computed using chain complexes involving tensor powers of $A$, and so, when $A$ is a general locally convex algebra, this construction will require a choice of a topological tensor product. Among the most frequently used topological tensor products defined on the class of locally convex topological vector spaces are the projective tensor product $\otimes_{\pi}$, the injective tensor product $\otimes_{\epsilon}$, and the inductive tensor product $\otimes_{i}$ and a priori each choice leads to a separate cyclic type homology theory (with further possibilities provided by completions of these products).

The algebra $C^{\infty}(M)$ of smooth functions on a compact manifold $M$, considered as a topological algebra in its standard seminorm topology, provides an important example of a Fréchet algebra that is also nuclear. Therefore, there is a unique tensor product which is compatible, in the sense of Grothendieck [12, I.89], with the algebraic tensor product $C^{\infty}(M) \otimes C^{\infty}(M)$. This topology was used, for example, by Connes in his computation of the cyclic cohomology of this algebra [3, Ch.II, Theorem 46].

When considering growth conditions on cochains, for instance as required by Connes' entire cyclic cohomology, the topological approach runs into an immediate difficulty. It has been well known since Grothendieck's Problème des Topologies, [12, p. 33] that in general there is no clear connection between bounded sets in the tensor product of two spaces, and the tensor products of bounded sets.

A possible circumvention of this problem rests on the properties of bounded sets, and this approach has been developed with great success by Meyer. We now sketch the main features.

If $X$ is a set, then a bornology on $X$ is a family $\mathcal{B}$ of subsets of $X$ which is closed with respect to taking subsets and finite unions. Elements of the family $\mathcal{B}$ will be called bounded sets, and a bornological space is a set equipped with a bornology. A base of a bornology $\mathcal{B}$ is a subfamily $\mathcal{B}_{0}$ of $\mathcal{B}$ with the property that any element of $\mathcal{B}$ is contained in some element of $\mathcal{B}_{0}$.

It is natural (and consistent with examples) to say that a map $f:(X, \mathcal{B}) \rightarrow\left(Y, \mathcal{B}^{\prime}\right)$ of bornological spaces is bounded if and only if for any $B \in \mathcal{B}, f(B) \in \mathcal{B}^{\prime}$. 
A bornology on a vector space $E$ is said to be compatible with the vector space structure iff the vector addition $E \times E \rightarrow E$ and multiplication by scalars $\mathbb{C} \times E \rightarrow E$ are bounded maps of bornological spaces.

When the space $E$ is a locally convex topological vector space, then its topology determines a canonical bornology, called the von Neumann bornology, which consists of all sets $S$ that are absorbed by all neighbourhoods of zero; this means that for every neighbourhood $\mathrm{U}$ of zero there exists a positive number $t$ such that $S \subseteq \mathrm{tU}$. More generally, one can equip the vector space $E$ with a bornology which does not arise from the topology on $E$. One consequence of this is that the class of bounded linear maps on $E$ will in general be different from the class of continuous maps.

From now on, all bornological spaces will be assumed to be vector spaces.

Let us now describe briefly the notion of bornological completion. A bornological vector space $E$ is called convex if its bornology has a base which consists of convex sets. If this is the case, we can assume that the base consists of balanced convex sets, which will be called discs. For a bounded disc $\mathrm{D}$ in $\mathrm{E}$, let $\mathrm{E}_{\mathrm{D}}$ denote the vector space generated by $\mathrm{D}$ and equipped with the seminorm given by the gauge of $D$. When $E$ is a Hausdorff space, $\mathrm{E}_{\mathrm{D}}$ is a normed space; when this space is complete, we call the disc $\mathrm{D}$ pre-complete. So, when $E$ is Hausdorff and $D$ pre-complete, $E_{D}$ is a Banach space. The spaces $E_{D}$ form an inductive system indexed by the directed family of bounded discs and $E$ is the direct limit of this system.

A complete bornological space is a bornological space E which admits a base consisting of pre-complete discs. A bornological space $E$ is complete iff it is the inductive limit of an injective inductive system of Banach spaces [18, Theorem A.4]. While every bornological space $E$ admits a bornological completion, this is less well behaved than the usual completion of a uniform space (see [18, Appendix A] for a full discussion).

Finally, we say that $A$ is a bornological algebra if and only if it is equipped with a vector space bornology with respect to which the product map $A \times A \rightarrow A$ is bounded; of course, a complete bornological algebra is one that is complete as a bornological vector space.

Proceeding by analogy with the topological tensor product, the bornological tensor product of two bornological spaces $\left(E_{1}, \mathcal{B}_{1}\right)$ and $\left(E_{2}, \mathcal{B}_{2}\right)$ is the algebraic tensor product $E_{1} \otimes E_{2}$ equipped with the bornology whose base consists of balanced convex hulls of sets of the form $B_{1} \otimes B_{2}$, where $B_{1} \in \mathcal{B}_{1}$ and $B_{2} \in \mathcal{B}_{2}$. This is precisely where we declare that bounded subsets of the tensor product arise from tensor products of bounded sets. The completed bornological tensor product $E_{1} \hat{\otimes} E_{2}$ is the bornological completion of $E_{1} \otimes E_{2}$ with respect to this bornology.

It is interesting to trace these constructions in particular examples, and Meyer provides a thorough discussion of how bornological tensor products of standard topological spaces relate to their topological counterparts. And so, the completed bornological tensor product of two Fréchet spaces equipped with the precompact bornology (i.e., the bornology generated by pre compact sets) is isomorphic to the completed projective tensor product of the two spaces [18, Theorem 2.29].

For the next example, let us first recall that a locally convex topological vector space $E$ is an LF-space if there exists an increasing sequence of subspaces $E_{n}, n \in \mathbb{N}$ with the property that each of the subspaces $E_{n}$ is Fréchet in the subspace topology, the union of all subspaces $E_{n}$ equals $E$ and $E$ is equipped with the finest topology so that all inclusion maps $\mathrm{E}_{\mathrm{n}} \rightarrow \mathrm{E}$ are continuous.

When $E_{1}$ and $E_{2}$ are nuclear ${ }^{1}$ LF-spaces regarded as bornological spaces with the von Neumann bornology then the completed bornological tensor product $\mathrm{E}_{1} \hat{\otimes}_{2}$ is isomorphic to the completed inductive tensor product of Grothendieck, see [18, Cor. 2.30, p. 15].

Let us now assume that $A$ is a complete bornological algebra. Then there is a natural bornology on $\Omega A$ whose base is given by balanced convex hulls of the sets $\mathrm{SdS} \ldots \mathrm{d} S$

\footnotetext{
${ }^{1}$ See [12, Chapter 2, Def. 4]
} 
and $\mathrm{d} S \ldots \mathrm{d} S$, where $S$ is an element of the bornology on $A$. Let us denote by $\Omega_{\mathrm{an}} A$ the completion of $\Omega A$ with respect to this bornology. This is again a $\mathbb{Z} / 2 \mathbb{Z}$-graded complex with the same differentials $b$ and $B$, which are now bounded maps. With this in hand, we define the analytic tensor algebra $\mathcal{T} A$ of $A$ to be the even part of the algebra $\Omega_{\mathrm{an}} A$, equipped with the Fedosov product; this algebra fits into the algebra extension

$$
0 \rightarrow \mathcal{J A} \rightarrow \mathcal{T A} \rightarrow \mathrm{A} \rightarrow 0
$$

Now the $\mathrm{X}$-complex of the algebra $\mathcal{T} A$ is defined using the construction outlined above.

Definition 3.1. The bivariant entire cyclic homology of a pair of bornological algebras $A$ and $\mathrm{B}$ is by definition

$$
\mathrm{HE}_{*}(A, B)=\mathrm{H}_{*}(\operatorname{Hom}(X(\mathcal{T} A), X(\mathcal{T B})) .
$$

where $\operatorname{Hom}(X(\mathcal{T A}), X(\mathcal{T B}))$ denotes the $\mathbb{Z} / 2 \mathbb{Z}$-graded complex of bounded linear maps from $X(\mathcal{T A})$ to $X(\mathcal{T} B)$. This complex is equipped with the differential $[\partial, \phi]=\partial \circ \phi-$ $(-1)^{\operatorname{deg} \phi} \phi \circ \partial$, where $\partial=b+B$ [7, p. 57][18, p. 37]. The resulting homology theory is $\mathbb{Z} / 2 \mathbb{Z}$-graded.

This construction is due to Meyer, who proved that HE is invariant with respect to differentiable homotopies whose first derivative is integrable. Moreover, the bivariant entire cyclic homology satisfies excision in both variables [7, Thm. 5.4][19]. More precisely, we have the following.

Theorem 3.2. Let $0 \rightarrow \mathrm{S} \rightarrow \mathrm{P} \rightarrow \mathrm{Q} \rightarrow \mathrm{O}$ be an extension of complete bornological algebras which admits a bounded linear section. Assume further that $\mathrm{A}$ is a complete bornological algebra. Then we have the following natural exact sequences of length six:

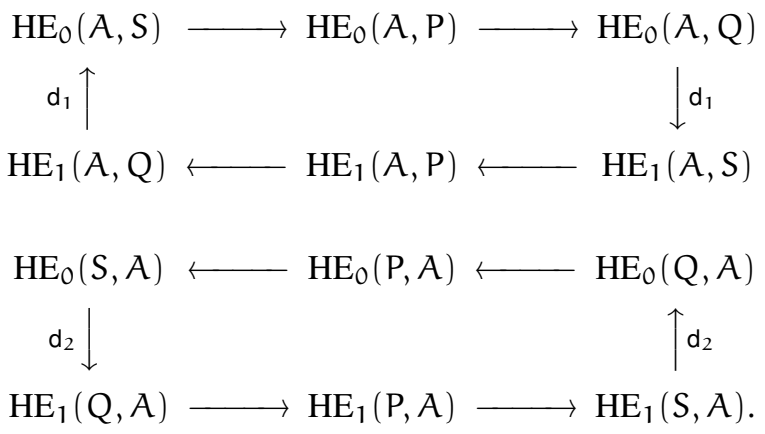

Meyer also proves that for any Banach algebra $A$, the bivariant entire cyclic homology $\operatorname{HE}_{*}(A, \mathbb{C})$ is the same as the entire cyclic cohomology $\operatorname{HE}^{*}(A)$ of $A$ as defined by Connes [20, 4.1]. This observation allows one to define the entire cyclic homology of $A$ by $\mathrm{HE}_{*}(A)=\mathrm{HE}_{*}(\mathbb{C}, A)$.

Another very important property of $\mathrm{HE}$ is the existence of the composition product, which is defined as in the case of bivariant periodic cyclic homology by composition of linear chain maps. For any three bornological algebras $A_{1}, A_{2}$ and $A_{3}$ there is a bilinear product

$$
\operatorname{HE}_{i}\left(A_{1}, A_{2}\right) \times \operatorname{HE}_{j}\left(A_{2}, A_{3}\right) \rightarrow \operatorname{HE}_{i+j}\left(A_{1}, A_{3}\right)
$$

given by $f \cdot g=g \circ f$. With these properties, the bivariant entire cyclic cohomology has formal properties similar to those of Kasparov's bivariant KK-theory. One crucial difference is that HE is only invariant with respect to homotopies which are 'sufficiently smooth'. Puschnigg's local bivariant cohomology does not have this restriction, as we shall explain later. 


\section{ENTIRE CYCLIC COHOMOLOGY OF SCHATtEN IDEALS}

To demonstrate the power of the algebraic formalism created by Meyer's extension of results of Cuntz and Quillen to bornological algebras, let us discuss briefly the computation of the entire cyclic cohomology of Schatten ideals carried out in [2]. It turns out that for $p \geq 1$, Schatten ideals display a remarkable stability with respect to HE.

Theorem 4.1. Let $\mathfrak{L}^{p}$ and $\mathfrak{L}^{\mathrm{q}}$ be two Schatten ideals, $1 \leq p<q$. Then the inclusion $\mathfrak{L}^{p} \rightarrow \mathfrak{L}^{\mathrm{q}}$ induces an invertible element in $\mathrm{HE}_{0}\left(\mathfrak{L}^{p}, \mathfrak{L}^{\mathrm{q}}\right)$. Consequently, the entire cyclic homology and cohomology of the two algebras are isomorphic:

$$
\operatorname{HE}^{\mathfrak{i}}\left(\mathfrak{L}^{\mathfrak{p}}\right)=\operatorname{HE}^{\mathfrak{i}}\left(\mathfrak{L}^{\mathfrak{q}}\right) ; \quad \operatorname{HE}_{\mathfrak{i}}\left(\mathfrak{L}^{\mathfrak{p}}\right)=\operatorname{HE}_{\mathfrak{i}}\left(\mathfrak{L}^{\mathfrak{q}}\right)
$$

for $i=0,1$.

In the context of algebraic periodic cyclic homology [7] as well as the kk-theory of Cuntz, this result was first proved by Cuntz [8].

For the proof one needs explicit formulae for the connecting homomorphisms in the commutative diagrams of Theorem 3.2. The construction outlined here relies on algebraic properties of bivariant cyclic homology and so works for all types of homology theories described in this paper. In particular, the same argument gives connecting homomorphisms in the algebraic excision theorem 2.2.

Let us consider the following extension $E$ of complete bornological algebras

$$
\mathrm{E}: \quad 0 \rightarrow \mathrm{S} \stackrel{\mathrm{i}}{\rightarrow} \mathrm{P} \stackrel{\mathrm{p}}{\rightarrow} \mathrm{Q} \rightarrow 0 .
$$

We shall assume that this sequence is split, i.e. there exists a bounded linear map s : $Q \rightarrow P$ which is a right inverse for the projection $p$.

The excision property of bivariant cyclic homology $\mathrm{HE}_{*}$ implies that there are two long exact sequences

$$
\begin{aligned}
& \rightarrow \mathrm{HE}_{*}(\mathrm{P}, \mathrm{S}) \rightarrow \mathrm{HE}_{*}(\mathrm{~S}, \mathrm{~S}) \stackrel{\delta_{1}}{\longrightarrow} \mathrm{HE}_{*+1}(\mathrm{Q}, \mathrm{S}) \rightarrow \\
& \rightarrow \mathrm{HE}_{*}(\mathrm{Q}, \mathrm{P}) \rightarrow \mathrm{HE}_{*}(\mathrm{Q}, \mathrm{Q}) \stackrel{\delta_{2}}{\longrightarrow} \mathrm{HE}_{*+1}(\mathrm{Q}, \mathrm{S}) \rightarrow .
\end{aligned}
$$

Let us denote by $1_{\mathrm{Q}}$ the class in $\mathrm{HE}_{0}(\mathrm{Q}, \mathrm{Q})$ induced by the identity map on the algebra $\mathrm{Q}$ and,similarly, let $1_{S}$ denote the class of the identity map on $S$.

Proposition 4.2. Let $\delta_{1}$ and $\delta_{2}$ denote the connecting homomorphisms in the two long exact sequences above. Then

$$
\delta_{1}\left(1_{S}\right)=-\delta_{2}\left(1_{Q}\right) \in \operatorname{HE}_{1}(Q, S) .
$$

This lemma can be regarded as an analogue of a result of Kassel [14, Lemme 2.2] concerning his bivariant cyclic cohomology, see also [9][7, Prop. 2.51, p.33].

This result can be used to provide formulae for connecting homomorphisms in the exact sequences of Theorem 3.2, which uses that the two sequences are natural. In the case of the sequence (1) in Theorem 3.2, this means that there exists a commutative diagram

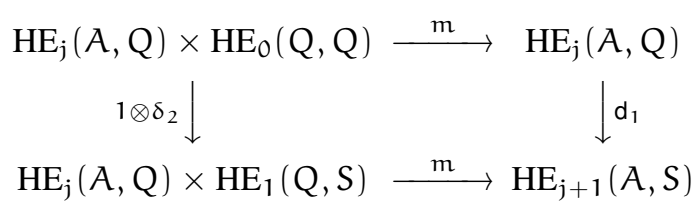

where $m$ denotes the product map and $d_{1}$ denotes the connecting homomorphism in the diagram (1) of Theorem 3.2, for $\mathfrak{j}=0,1$.

Taking into account the usual sign convention we have that

$$
\mathrm{d}_{1}(\phi \cdot \psi)=\mathrm{m}\left(1 \otimes \delta_{2}\right)(\phi \otimes \psi)=\mathrm{m}\left((-1)^{\operatorname{deg}(\phi)}\left(\phi \otimes \delta_{2}(\psi)\right)=(-1)^{\operatorname{deg}(\phi)} \phi \cdot \delta_{2}(\psi)\right.
$$


for $\phi \in \mathrm{HE}_{\mathrm{j}}(A, \mathrm{Q})$ and $\psi \in \mathrm{HE}_{0}(\mathrm{Q}, \mathrm{Q})$. Hence

$$
\mathrm{d}_{1}(\phi)=\mathrm{d}_{1}\left(\phi \cdot 1_{\mathrm{Q}}\right)=(-1)^{\operatorname{deg}(\phi)} \phi \cdot \delta_{2}\left(1_{\mathrm{Q}}\right)
$$

Similarly, we obtain a formula for the connecting homomorphism $d_{2}$ in the exact sequence (2). In this case, the naturality of this sequence implies that there exists a commutative diagram

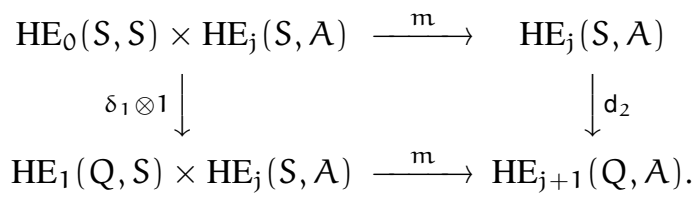

Hence, for $\phi \in \mathrm{HE}_{0}(S, S)$ and $\phi \in \mathrm{HE}_{j}(S, A)$ we have

$$
\mathrm{d}_{2}(\phi \cdot \psi)=\mathrm{m}\left(\delta_{1} \otimes 1\right)(\phi \otimes \psi)=\delta_{1}(\phi) \cdot \psi
$$

Thus

$$
\mathrm{d}_{2}(\psi)=\mathrm{d}_{2}\left(1_{\mathrm{S}} \cdot \psi\right)=\delta_{1}\left(1_{S}\right) \cdot \psi .
$$

This kind of consideration allows one to define a Chern class of the extension $E$.

Proposition 4.3. Let us denote by $\mathrm{ch}(\mathrm{E})$ the class $-\delta_{1}\left(1_{\mathrm{S}}\right)=\delta_{2}\left(1_{\mathrm{Q}}\right)$ of the extension $\mathrm{E}$. Then the connecting homomorphism $\mathrm{d}_{1}$ in the exact sequence (1) of Theorem 3.2 sends $\phi \in \mathrm{HE}_{\mathrm{j}}(\mathrm{A}, \mathrm{Q})$ to $(-1)^{\operatorname{deg}(\phi)} \phi \cdot \operatorname{ch}(\mathrm{E}) \in \mathrm{HE}_{\mathrm{j}+1}(\mathrm{~A}, \mathrm{~S})$. The connecting homomorphism $\mathrm{d}_{2}$ in the sequence (2) of Theorem 3.2 sends $\psi \in \mathrm{HE}_{\mathrm{j}}(\mathrm{S}, A)$ to $\operatorname{ch}(\mathrm{E}) \cdot \psi \in \mathrm{HE}_{\mathrm{j}+1}(\mathrm{Q}, A)$.

This implies, as in [14][9], the following.

Corollary 4.4. If the algebra $\mathrm{P}$ in the extension $\mathrm{E}$ is $\mathrm{HE}$-equivalent to 0 , which means that $\mathrm{HE}_{*}(\mathrm{~A}, \mathrm{P})=\mathrm{HE}_{*}(\mathrm{P}, \mathrm{A})=0$ for any bornological algebra $\mathrm{A}$, then $\mathrm{ch}(\mathrm{E})$ is an invertible element in $\mathrm{HE}_{1}(\mathrm{Q}, \mathrm{S})$.

Proof. Let us put $A=S$ in the sequence (1) and then $A=Q$ in the sequence (2) of Theorem 3.2. Since the terms containing the algebra $P$ are zero, we see that the connecting homomorphisms $\mathrm{d}_{1}$ and $\mathrm{d}_{2}$ are now isomorphisms. In particular, there exists $\eta_{1} \in \operatorname{HE}_{1}(S, Q)$ such that $d_{1}\left(\eta_{1}\right)=1_{S} \in \operatorname{HE}_{0}(S, S)$. Similarly, there exists $\eta_{2} \in \operatorname{HE}_{1}(S, Q)$ such that $d_{2}\left(\eta_{2}\right)=1_{Q} \in H_{0}(Q, Q)$. But we have just established that

and that

$$
\mathrm{d}_{1}\left(\eta_{1}\right)=\eta_{1} \cdot \operatorname{ch}(E)=1_{s}
$$

$$
\mathrm{d}_{2}\left(\eta_{2}\right)=\operatorname{ch}(E) \cdot \eta_{2}=1_{\mathrm{Q}}
$$

This implies that $\eta_{1}=\eta_{2}$. Indeed,

$$
\eta_{1}=\eta_{1} \cdot 1_{\mathrm{Q}}=\eta_{1} \cdot \operatorname{ch}(E) \cdot \eta_{2}=1_{S} \cdot \eta_{2}=\eta_{2} \text {. }
$$

Thus $\eta=\eta_{1}=\eta_{2} \in \operatorname{HE}_{1}(S, Q)$ is the inverse of $\operatorname{ch}(E) \in \mathrm{HE}_{1}(\mathrm{Q}, S)$.

To apply these results to computing the entire cyclic cohomology of Schatten classes, one can adapt a method of Cuntz [8, Satz 6.12][7], who used it to prove an analogous result in his kk theory and periodic cyclic homology.

Proposition 4.5. Let us assume that for two complete bornological algebras $\mathrm{A}$ and $\mathrm{B}$ there are maps

$$
\begin{array}{ll}
\alpha: & B \hookrightarrow A \\
\beta: & A \otimes A \rightarrow B
\end{array}
$$

such that the composition $\alpha \circ \beta$ is identical to the product map on $A$, whereas $\beta \circ(\alpha \otimes \alpha)$. is the product on $\mathrm{B}$. Then the element $[\alpha]$ of $\mathrm{HE}_{0}(\mathrm{~B}, \mathrm{~A})$ is invertible. This implies that $\mathrm{HE}^{*}(A) \simeq \mathrm{HE}^{*}(\mathrm{~B})$ and $\mathrm{HE}_{*}(\mathrm{~B}) \simeq \mathrm{HE}_{*}(\mathrm{~A})$. 
The proof of Theorem 4.1 is concluded by applying these results in the case when $\mathrm{B}=\mathfrak{L}^{p}$ and $A=\mathfrak{L}^{\mathrm{q}}$, where $p \leq q \leq 2 p$. The map $\alpha$ of the previous statements is obtained from the continuous inclusion $\mathfrak{L}^{p} \rightarrow \mathfrak{L}^{q}$ and the map $\beta$ from the multiplication map $\mathfrak{L}^{\mathrm{q}} \hat{\otimes} \mathfrak{L}^{\mathrm{q}} \rightarrow \mathfrak{L}^{\mathrm{p}}$.

\section{Continuous trace Algebras}

The computation of the entire cyclic cohomology of Schatten ideals enabled Mathai and Stevenson to establish that entire and periodic cyclic cohomology are isomorphic on a large class of algebras [17] defined in the following Theorem.

Theorem 5.1. Let $\mathcal{C}$ be the smallest class of Fréchet algebras satisfying the following properties:

(i) The class $\mathcal{C}$ contains $\mathbb{C}$ and all Schatten ideals $\mathcal{L}^{p}$ for all $\mathrm{p} \geq 1$;

(ii) $\mathcal{C}$ is closed under smooth homotopy equivalence;

(iii) If in an extension admitting a continuous section, two of the algebras belong to $\mathcal{C}$, then so does the third.

Then for every algebra $\mathcal{A} \in \mathcal{C}$, the natural map between continuous periodic homology and entire cyclic homology induces an isomorphism

$$
\mathrm{HE}_{*}(\mathcal{A}) \cong \mathrm{HP}_{*}(\mathcal{A}),
$$

where $\mathcal{A}$ is equipped with the precompact bornology.

The proof rests on an observation that the class $\mathcal{C}^{\prime}$ of Fréchet algebras (equipped with the canonical complete bornology) for which the isomorphism (3) holds satisfies the three conditions stated in the theorem. Indeed, the category $\mathcal{C}^{\prime}$ contains $\mathbb{C}$. It also contains the Schatten ideals, which follows from Theorem 4.1. Since both entire and periodic cyclic homology are invariant with respect to smooth homotopies, the category $\mathcal{C}^{\prime}$ is closed under smooth homotopy equivalence. Let us then consider an extension of algebras of Fréchet algebras admitting a continuous section in which two of the algebras belong to the category $\mathrm{C}^{\prime}$. Then applying excision in both theories we will create two exact sequences of length six connected by natural transfer maps from continuous periodic homology to entire cyclic homology. By definition of $\mathcal{C}^{\prime}$, these maps will be isomorphisms for the two algebras in our extension that belong to $\mathcal{C}^{\prime}$. By the five lemma, the transfer maps corresponding to the third algebra will also be isomorphisms, and so this algebra will also belong to $\mathcal{C}^{\prime}$. This implies that $\mathcal{C} \subseteq \mathcal{C}^{\prime}$ and so the isomorphism (3) holds for every algebra in the category $\mathcal{C}$.

An interesting application of this theorem is the computation of entire cyclic homology for a class of continuous trace $C^{*}$-algebras. Let $A$ be a continuous trace $C^{*}$-algebra whose spectrum is a smooth compact manifold $M$. A key result, due to Dixmier and Duady, identifies an algebra $A$ of this form with the algebra $A=C(M, \mathcal{K}(P))$ of continuous sections of a smooth locally trivial bundle $\mathcal{K}(\mathrm{P})=\mathrm{P} \times_{\mathrm{pu}} \mathcal{K}$, where the fibre $\mathcal{K}$ is the algebra of compact operators on a separable Hilbert space, which is an associated bundle of a principal PU bundle $\mathrm{P}$ on $\mathrm{M}$. Here PU denotes the projective unitary group of a Hilbert space.

As is well known, algebras $A$ of this type are classified, up to isomorphism, by their Dixmier-Douady invariant $\delta(P) \in H^{3}(M, \mathbb{Z})$. For every $p \geq 1$, such an algebra $A$ contains a canonical dense, smooth $*$-subalgebra $\mathcal{A}_{\mathrm{p}}=\mathrm{C}^{\infty}\left(\mathrm{M}, \mathcal{L}^{\mathrm{p}}(\mathrm{P})\right)$, which is the algebra of smooth sections of a canonical sub-bundle $\mathcal{L}^{p}(\mathrm{P})=\mathrm{P} \times{ }_{\mathrm{PU}} \mathcal{L}^{\mathrm{p}}$ of $\mathcal{K}(\mathrm{P})$, with fibre $\mathcal{L}^{\mathrm{p}}$ and structure group PU.

Theorem 5.2. Let $M$ be a smooth connected compact manifold equipped with a principal $\mathrm{PU}$-bundle $\mathrm{P} \rightarrow \mathrm{M}$. Consider the nuclear Fréchet algebra $\mathcal{A}_{\mathrm{p}}$ of smooth sections of the bundle $\mathcal{L}^{\mathrm{p}}(\mathrm{P})=\mathrm{P} \times \mathrm{Pu} \mathcal{L}^{\mathrm{p}}$. Then for all $\mathrm{p} \geq 1$ there are isomorphisms

$$
\operatorname{HE}_{*}\left(\mathcal{A}_{\mathfrak{p}}\right) \cong \operatorname{HP}_{*}\left(\mathcal{A}_{\mathfrak{p}}\right) \text {. }
$$


When this conclusion is combined with the main result of [16] then we have the following complete description of the entire cyclic cohomology of the algebras $\mathcal{A}_{\mathrm{p}}$.

Corollary 5.3. [17, Cor. 3.3] The entire cyclic homology $\operatorname{HE}_{*}\left(\mathcal{A}_{\mathfrak{p}}\right)$ is isomorphic to the twisted de Rham cohomology $\mathrm{H}^{*}(\mathrm{M}, \mathrm{c}(\mathrm{P}))$ for some closed 3-form on $\mathrm{M}$ such that $(1 / 2 \pi i) c(P)$ represents the image of the Dixmier-Douady invariant $\delta(P)$ in cohomology with real coefficients.

\section{THE LOCAL BIVARIANT CYCLIC COHOMOLOGY}

It is worth stressing, thanks to the work of Cuntz and Quillen, that the different types of cyclic homology theories of an algebra $A$ arise from a complex that is built using various completions of canonical tensor algebra $\mathcal{T} A$ associated with $A$. In the case of Cuntz's and Quillen's own work, it was the adic completion with respect to powers of the ideal spanned by differential forms of degree at least one. Meyer's study of entire cyclic cohomology used the bornological completion. We now come to another important version of cyclic type homology: Puschnigg's local bivariant cohomology.

This theory is developed for the so called Ind-algebras with supports. The first notion comes from category theory, where for a category $\mathcal{C}$ one constructs a category Ind $\mathcal{C}$ of formal inductive limits. In essence, this means considering inductive systems of objects in $\mathcal{C}$, with morphisms defined using a natural notion of morphism of two inductive systems. An important reason for doing it in this way is that an inductive limit of of a system of objects belonging to one category may not necessarily belong to that category; Banach spaces provide an example. So an Ind-algebra $\mathcal{A}$ can be thought of as an inductive system of algebras $A_{i}$ from some category $\mathcal{C}$ of algebras, where $i \in$ I runs through an indexing set I. Now a support of an Ind-algebra is a family $\mathcal{S}$ of non-empty subsets $S_{i}$ of $A_{i}$, which is directed in the sense that for all $S_{i}, S_{j} \in \mathcal{S}$ there exists $S_{k} \in \mathcal{S}$ such that both $S_{i}$ and $S_{j}$ are contained in $S_{k}$. We further assume that $\mathcal{S}$ contains the multiplicative closure of the sets $S_{i}$. Moreover, if all algebras $A_{i}$ are Fréchet algebras, we assume that the set $S_{i}$ is bounded in $A_{i}$, for all $i \in I$. There is a natural notion of support-preserving maps of Ind-algebras, which are maps of inductive systems $\phi: \mathcal{A} \rightarrow \mathcal{A}^{\prime}$ so that for every $i \in \mathrm{I}, \phi\left(S_{i}\right) \subseteq S_{i}^{\prime}$.

An admissible Fréchet algebra is a Fréchet algebra $A$ with the property that there exists an open neighbourhood $U$ of zero in $A$ such that the multiplicative closure of any compact subset of $U$ is relatively compact in $A$. This is equivalent to saying that the family of compact subsets of $U$ provides a system of supports $\mathcal{S}_{c}$ for $A$. Among examples of admissible Fréchet algebras are Banach algebras, and many Fréchet algebras that are smooth, dense, and holomorphically closed subalgebras of Banach algebras.

One needs to extend the formalism of tensor algebras and the X-complex to Ind-algebras, and this is a main part of Puschnigg's foundational work. A particular technical point to be aware of is that a system of supports of an Ind-algebra $\mathcal{A}$ creates a system of supports for the tensor Ind-algebra $\mathcal{T}_{\text {Ind }} \mathcal{A}$. Once we form the analogue of the $\mathrm{X}$ complex for algebras of this type, we will require that any map of complexes preserve the supports. With this proviso we define the bivariant local cyclic homology of two Ind-algebras $\left(\mathcal{A}, \mathcal{S}_{\mathcal{A}}\right)$ and $\left(\mathcal{B}, \mathcal{S}_{\mathcal{B}}\right)$ with supports by

$$
\mathrm{HL}_{*}(\mathcal{A}, \mathcal{B})=\mathrm{H}_{*}\left(\operatorname{Hom}\left(\mathrm{X}\left(\mathcal{T}_{\text {Ind }} \mathcal{A}\right), \mathrm{X}\left(\mathcal{T}_{\text {Ind }} \mathcal{B}\right)\right)\right) .
$$

Remember that in the case of Meyer's analytic cohomology, we were interested in bounded maps, which preserve the bornologies of the algebras in question; this clearly is an example of Puschnigg's support condition when Meyer's and Puschnigg's theories are defined on the same class of algebras. In fact, Puschnigg proves the following.

Theorem 6.1. [27, Prop. 3.21, Lemma 3.22]

(1) Let A be a Banach algebra. Then its cyclic cohomology with bounded supports is identical to Connes' entire cyclic cohomology; 
(2) Let A and B be admissible Fréchet algebras with compact supports. Then local bivariant cyclic cohomology with compact supports is the same as Meyer's analytic bivariant cohomology defined with respect to the compact bornology.

One advantage of local cyclic cohomology is that it allows a larger class of algebras as its arguments, and in particular it works for $\mathrm{C}^{*}$-algebras, and in this case it has the same formal properties as the KK-theory of Kasparov: it is stable, satisfies excision, and is invariant with respect to continuous homotopies. The universal property of KK-theory now guarantees the existence of the Chern character, as in the theorem below. This is an important conclusion of a long effort to construct the Chern character in cyclic type cohomology theories.

Theorem 6.2. [25, Theorem 6.3] There exists a natural transformation of bifunctors on the category of separable $\mathrm{C}^{*}$-algebras

$$
\operatorname{ch}: \mathrm{KK}_{*}(\mathrm{~A}, \mathrm{~B}) \rightarrow \mathrm{HL}_{*}(\mathrm{~A}, \mathrm{~B})
$$

from Kasparov's bivariant KK-theory to bivariant local cyclic cohomology, called the bivariant Chern-Connes character.

The bivariant Chern character is compatible with long exact sequences (in both variables) arising from an extension $\mathrm{O} \rightarrow \mathrm{I} \rightarrow \mathrm{A} \rightarrow \mathrm{B} \rightarrow 0$ of separable $\mathrm{C}^{*}$-algebras with a completely positive section. It is also compatible with the Kasparov product in KK-theory and the composition product in $\mathrm{HL}$.

We now give two applications of local cyclic cohomology. The first is to the computation of the entire cyclic cohomology of the algebra $C^{\infty}(M)$ of smooth functions on a manifold $M$. To appreciate this result, it is necessary to remark that an important motivation for the introduction of Connes' entire cyclic cohomology was that it is the right theory in the context of infinite dimensional non commutative spaces. So in the case of finite dimensional spaces, for instance finite dimensional manifolds, the entire cyclic cohomology should be the same as the periodic cyclic cohomology, which was devised for finite dimensional spaces. This simple-to-state problem turned out to be very difficult to solve and required the whole power of local cyclic cohomology. We have the following.

Theorem 6.3. [25, Theorem 6.2] Let $M$ be a smooth, compact manifold with (possibly empty) boundary and let $\mathrm{C}^{\infty}(\mathrm{M})$ be the nuclear admissible Fréchet algebra of smooth functions on $\mathrm{M}$. Then there are natural isomorphisms

$$
\begin{aligned}
& \operatorname{HE}_{*}\left(\mathcal{C}^{\infty}(M)\right) \cong \mathrm{HP}_{*}\left(\mathcal{C}^{\infty}(M)\right) \cong \mathrm{H}\left(M, \mathrm{HP}_{*}(\mathbb{C})\right) \\
& \operatorname{HE}^{*}\left(\mathrm{C}^{\infty}(M)\right) \cong \operatorname{HP}^{*}\left(\complement^{\infty}(M)\right) \cong \mathrm{H}_{*}\left(M, \operatorname{HP}^{*}(\mathbb{C})\right)
\end{aligned}
$$

of homology and cohomology groups.

The proof, in outline, goes as follows. Let $\mathrm{N}$ be a codimension one submanifold without boundary which does not intersect $\partial M$, and which has the property that the tangent bundle of $N$ is trivial. There are two algebras associated with this data: $C^{\infty}(M, N)$ is the algebra of smooth functions on $M$ that vanish on $N$, and $C_{0}^{\infty}(M, N)$ is the subalgebra of $C^{\infty}(M, N)$ consisting of all smooth functions $f$ on $M$ such that $f$ and all its derivatives vanish on $N$. It turns out that the inclusion $C_{0}^{\infty}(M, N) \hookrightarrow C^{\infty}(M, N)$ is a smooth homotopy equivalence.

We now set up an induction argument with respect to the dimension of $M$, aiming to show that $C^{\infty}(M)$ belongs to the class $\mathcal{C}$ described in Theorem 5.1 for any smooth manifold $M$. So let us assume that this was proved for all manifolds with potentially nonempty boundary whose dimension is smaller than $\operatorname{dim} M$. Let us choose a Morse function $f: M \rightarrow \mathbb{R}_{+}$which has finitely many critical points $x_{1}, \ldots, x_{k}$ so that no two lie on the same level surface of $f$. We assume that 0 is a regular value and that $\partial M=f^{-1}(0)$. Let $t_{0}=0$ and choose real numbers $t_{1}, \ldots, t_{k}$ such that $t_{i-1}<f\left(x_{i}\right)<t_{i}$, for $i=$ 
$1, \ldots, k$ and let $N_{i}=f^{-1}\left(t_{i}\right)$. This leads to the extension of nuclear, admissible Fréchet algebras

$$
\mathrm{E}: \quad 0 \rightarrow \mathrm{C}^{\infty}\left(\mathrm{M}, \coprod_{\mathrm{i}}\right) \rightarrow \mathrm{C}^{\infty}(\mathrm{M}) \rightarrow \mathrm{C}^{\infty}\left(\coprod_{\mathrm{i}}\right) \rightarrow 0
$$

which is split by a bounded linear section. By the induction hypothesis, the algebra $\mathrm{C}^{\infty}\left(\coprod \mathrm{N}_{i}\right)$ belongs to the category $\mathrm{C}$, so it is sufficient to prove that $\mathrm{C}^{\infty}\left(M, \coprod \mathrm{N}_{i}\right) \in \mathcal{C}$.

By the first step, there are smooth homotopy equivalences

$$
C^{\infty}\left(M, \amalg N_{i}\right) \sim C_{0}^{\infty}\left(M, \amalg N_{i}\right) \cong \bigoplus C_{0}^{\infty}\left(f^{-1}\left(\left[t_{i}, t_{i+1}\right]\right), N_{i} \coprod N_{i+1}\right) .
$$

Hence the result will follow once it is verified that each of the summands on the right hand side is an element of $\mathcal{C}$. This is achieved by using a handle decomposition of $M$ which gives a homotopy equivalence

$$
C_{0}^{\infty}\left(f^{-1}\left(\left[t_{i}, t_{i+1}\right]\right), N_{i} \coprod_{i+1}\right) \sim C^{\infty}\left(D^{n_{i}} \times S^{m_{i}}, \partial D^{n_{i}} \times D^{m_{i}}\right),
$$

where $D^{n_{i}}$ denotes a disc and $S^{m_{j}}$ is a sphere of the indicated dimension. The algebra on the right is in turn homotopy equivalent to the algebra

$$
C^{\infty}\left(D^{n_{i}} \times S^{m_{i}}, \partial D^{n_{i}} \times S^{m_{i}}\right),
$$

where $n_{i}+m_{i}=\operatorname{dim} M, n_{i}>0$. We now have an algebra extension

$$
0 \rightarrow C^{\infty}\left(D^{n_{i}} \times S^{m_{i}}, \partial D^{n_{i}} \times S^{m_{i}}\right) \rightarrow C^{\infty}\left(D^{n_{i}} \times S^{m_{i}}\right) \rightarrow C^{\infty}\left(\partial D^{n_{i}} \times S^{m_{i}}\right) \rightarrow 0 .
$$

The algebra $C^{\infty}\left(D^{n_{i}} \times S^{m_{i}}\right)$ is homotopy equivalent to $C^{\infty}\left(S^{m_{i}}\right)$, and so is an element of $\mathcal{C}$ by the induction hypothesis. By the same hypothesis, we have that the algebra on the right is in $\mathcal{C}$, which proves that the algebra on the left is an object of the category $\mathcal{C}$ as well. But this implies that the two algebras on either end of the extension $E$ are objects of the category $\mathcal{C}$ and thus so is $\mathcal{C}^{\infty}(M)$.

\section{THE KADISON-KAPLANSKY CONJECTURE FOR HYPERBOLIC GROUPS}

Our second example of an application of the local cyclic theory is to the KadisonKaplansky conjecture. Let $\Gamma$ be a discrete torsion-free group. Then the conjecture asserts that there are no non-trivial idempotents in the reduced $C^{*}$-algebra $C_{r}^{*}(\Gamma)$. This conjecture, long a focus of some really exciting research, is now one of the corollaries of the Baum-Connes conjecture.

Let $\mathrm{D}$ be an elliptic operator acting on the space of sections of a vector bundle over a smooth compact manifold $M$ without boundary. Then $D$ has a lift to a $\Gamma$-equivariant operator $\tilde{\mathrm{D}}$ on a $\Gamma$-covering $\widetilde{M}$ of $M$. Kasparov assigns to this data a topological index $\operatorname{Ind}_{\mathrm{t}}(\tilde{\mathrm{D}}) \in \mathrm{K}_{0}^{\mathrm{top}}(\mathrm{B} \Gamma)$, an analytic index $\operatorname{Ind}_{\mathrm{a}}(\tilde{\mathrm{D}}) \in \mathrm{K}_{0}\left(\mathrm{C}_{\mathrm{r}}^{*}(\Gamma)\right)$ and an assembly map

such that $\mu\left(\operatorname{Ind}_{a}(\tilde{D})\right)=\operatorname{Ind}_{a}(\tilde{D})$.

$$
\mu: \mathrm{K}_{*}^{\text {top }}(\mathrm{B} \Gamma) \rightarrow \mathrm{K}_{*}\left(\mathrm{C}_{\mathrm{r}}^{*}(\Gamma)\right)
$$

The Atiyah-Singer index theorem states that the topological and the analytic index are equal, while the Baum-Connes conjecture extends this statement to a hypothesis that the assembly map is an isomorphism. We should note that the Baum-Connes conjecture is stated for a far greater class of groups than the one considered here.

To deduce the Kadison-Kaplansky conjecture for a torsion-free group which satisfies the Baum-Connes conjecture, let $e$ be an idempotent in $\mathrm{C}_{\mathrm{r}}^{*}(\Gamma)$. Then e determines a class $[e] \in$ $\mathrm{K}_{0}\left(\mathrm{C}_{\mathrm{r}}^{*}(\Gamma)\right.$ which is the image, via the assembly map, of the topological index $\operatorname{Ind}_{t}(\tilde{\mathrm{D}})$, where $\tilde{D}$ is a $\Gamma$-invariant elliptic operator on the $\Gamma$-cover $\tilde{M}$ of the manifold $M$. Let $\tau$ be the canonical faithful trace on $\mathrm{C}_{\mathrm{r}}^{*}(\Gamma)$. It is well known that it extends to a map $\tau$ : $\mathrm{K}_{0}\left(\mathrm{C}_{\mathrm{r}}^{*}(\Gamma)\right) \rightarrow \mathbb{C}$. Atiyah's $\mathrm{L}^{2}$-index theorem then states that trace $\tau(e)=\tau\left(\operatorname{Ind}_{\mathrm{a}}(\tilde{\mathrm{D}})\right)=$ $\operatorname{Ind}(\mathrm{D})$, and so is an integer. The integrality of the trace of an arbitrary projection in $\mathrm{C}_{\mathrm{r}}^{*}(\Gamma)$ is equivalent to the Kadison-Kaplansky conjecture.

Mineyev and $\mathrm{Yu}$ [21] proved the Baum-Connes conjecture for hyperbolic groups using the strategy proposed by Lafforgue [15], which then implies the Kadison-Kaplansky 
conjecture. Essentially at the same time, Puschnigg presented a proof of the KadisonKaplansky conjecture for this class of groups [26]. His proof provides an excellent illustration of the power of cyclic homology when combined with KK-theory, and so it is worth to recall it in the present context.

Connes and Moscovici [4] proposed a strategy for proving the Baum-Connes conjecture which relies on the existence of a suitable Chern character with values in periodic cyclic cohomology. In Puschnigg's case, this can be stated as follows. Let $\mathfrak{a}(\Gamma)$ denote a Banach algebra completion of the group ring $\mathbb{C} \Gamma$ which is a subalgebra of the reduced $\mathrm{C}^{*}$-algebra $\mathrm{C}_{\mathrm{r}}^{*}(\Gamma)$. Then there is the following commutative diagram

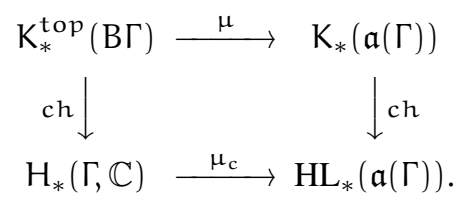

Here $\mathrm{H}_{*}(\Gamma, \mathbb{C})$ denotes the periodised group cohomology of $\Gamma$ with complex coefficients. Let us concentrate on the cohomological assembly map $\mu_{c}$ along the bottom row of the above diagram.

There is a natural adjoint action of $\Gamma$ on the complex defining the local cyclic cohomology with respect to which it decomposes as a direct sum of subcomplexes, indexed by conjugacy classes of $\Gamma$. The induced action creates a decomposition on cohomology, and in fact it turns out that $\mathrm{HL}_{*}(\mathfrak{a}(\Gamma))$ is a direct sum of the homogeneous part $\mathrm{HL}_{*}(\mathfrak{a}(\Gamma))$, corresponding to the identity element, and the inhomogeneous part, indexed by the nonidentity elements of $\Gamma$. The first important step in the proof is the fact that $\mu_{c}$ determines an isomorphism onto the homogeneous part of $\mathrm{HL}_{*}(\mathfrak{a}(\Gamma))$ :

$$
\mu_{\mathrm{c}}: \mathrm{H}_{*}(\Gamma, \mathbb{C}) \stackrel{\cong}{\longrightarrow} \mathrm{HL}_{*}(\mathfrak{a}(\Gamma))_{\text {hom }} .
$$

Moreover, Puschnigg's local cyclic cohomology possesses the following important stability property. When $\mathfrak{a}(\Gamma)$ is a large enough 'good' completion of $\mathbb{C} \Gamma$ in $C_{r}^{*}(\Gamma)$ in the sense of Lafforgue [15], then

$$
\mathrm{HL}_{*}\left(\ell^{1}(\Gamma)\right)_{\text {hom }} \cong \mathrm{HL}_{*}(\mathfrak{a}(\mathrm{L}))_{\text {hom }} .
$$

In fact this result holds also for crossed products. Moreover, we have the following.

Theorem 7.1. [26, Theorem 3.11] Let $\Gamma$ be a word hyperbolic group. Then there is an isomorphism

$$
\mathrm{HL}_{*}\left(\ell^{1}(\Gamma)\right)_{\mathrm{hom}} \cong \mathrm{H}_{*}(\Gamma, \mathbb{C})
$$

between the homogeneous part of $\mathrm{HL}_{*}\left(\ell^{1}(\Gamma)\right)$ and the periodised group homology $\mathrm{H}_{*}(\Gamma, \mathbb{C})$.

In Kasparov's approach to the Baum-Connes conjecture one aims at constructing an element $\gamma$ which is an idempotent in the ring $\mathrm{KK}^{\Gamma}(\mathbb{C}, \mathbb{C})$. The descent homomorphism

$$
\mathrm{j}_{\mathrm{r}}: \mathrm{KK}^{\Gamma}(\mathbb{C}, \mathbb{C}) \rightarrow \mathrm{KK}\left(\mathrm{C}_{\mathrm{r}}^{*}(\Gamma), \mathrm{C}_{\mathrm{r}}^{*}(\Gamma)\right)
$$

maps $\gamma$ to an element $j_{r}(\gamma)$, which acts as a projection $K_{*}\left(C_{r}^{*}(\Gamma)\right) \rightarrow \mu\left(K_{*}^{\text {top }}(B \Gamma)\right)$ onto the image of the topological assembly map. It follows that when $\gamma=1$, the assembly map is an isomorphism and the Baum-Connes conjecture holds for $\Gamma$. While in general $\gamma \neq 1$, Puschnigg shows that the existence of the $\gamma$ element combined with his computation of the local cyclic homology of $\ell^{1}(\Gamma)$ is sufficient to prove the Kadison-Kaplansky conjecture.

Theorem 7.2. [26, Theorem 5.6] Let $\Gamma$ be a torsion-free discrete group such that

(1) The classifying space $\mathrm{B} Г$ has the homotopy type of a finite simplicial complex;

(2) there exists a sufficiently large good completion $\mathfrak{a}(\Gamma)$ of $\mathbb{C} \Gamma$ in $\mathrm{C}_{\mathrm{r}}^{*}(\Gamma)$ which is stable under holomorphic functional calculus in $\mathrm{C}_{\mathrm{r}}^{*}(\Gamma)$;

(3) there exists a $\gamma$-element $\gamma \in \mathrm{KK}^{\Gamma}(\mathbb{C}, \mathbb{C})$;

(4) $\mathrm{HL}_{*}\left(\ell^{1}(\Gamma)\right)_{\text {hom }} \cong \mathrm{H}_{*}(\Gamma, \mathbb{C})$. 
Then the Kadison-Kaplansky conjecture holds for $\Gamma$. In particular, the Kadison-Kaplansky conjecture holds for any torsion-free word hyperbolic group.

The proof proceeds as follows. We select a sufficiently large good completion $\mathfrak{a}(\Gamma)$ of $\mathbb{C} \Gamma$ which is closed under holomorphic functional calculus in $\mathrm{C}_{\mathrm{r}}^{*}(\Gamma)$. The canonical trace $\tau$ on $C_{r}^{*}(\Gamma)$ vanishes on the inhomogeneous part of $C_{r}^{*}(\Gamma)$ and its restriction to $\mathfrak{a}(\Gamma)$ gives rise to a homogeneous local cyclic cocycle $[\tau] \in \mathrm{HL}^{0}(\mathfrak{a}(\Gamma))_{\text {hom. }}$. The Chern character is compatible with the decomposition into homogeneous and inhomogeneous parts and in particular it transports the inhomogeneous part $j_{\mathrm{r}}(1-\gamma)\left(K_{*}(\mathfrak{a}(\Gamma))\right.$ to the inhomogenous part of $H_{*}(\mathfrak{a}(\Gamma))$. This implies that the cocycle $[\tau]$ vanishes on $\operatorname{ch}\left(\mathfrak{j}_{r}(1-\gamma)\left(K_{*}(\mathfrak{a}(\Gamma))\right.\right.$. Moreover, using formula (4), the homogeneous component of the Chern character lands in the image of the homological assembly map. This is crucial, as one can now use the AtiyahSinger argument to show that the trace of any idempotent in $C_{r}^{*}(\Gamma)$ is an integer, which implies the Kadison-Kaplansky conjecture. It is important to stress that this argument uses only the existence of the $\gamma$ element, and not the veracity of the Baum-Connes conjecture for $\Gamma$. It is the vanishing of the canonical trace on the inhomogeneous part of HL that allows one to reduce the consideration to the image of the assembly map. Finally, Puschnigg shows that conditions of this theorem are satisfied by any torsion-free word hyperbolic group $\Gamma$, which concludes his proof of the Kadison-Kaplansky conjecture.

\section{THE BAUM-CONNES CONJECTURE FOR $p$-ADIC GROUPS}

In this final section, we would like to present a particular direction in the study of the Baum-Connes conjecture that has seen interesting developments based on new results in cyclic cohomology.

In his paper [29], Solleveld studies the category $\mathcal{C}$ consisting of those $m$-algebras $A$ for which the Chern character (as defined above) induces an isomorphism

$$
\operatorname{ch} \otimes \mathrm{id}: \mathrm{K}_{*}(A) \otimes \mathbb{C} \rightarrow \operatorname{HP}_{*}(A) .
$$

Using excision and other formal properties of cyclic type homology theories he proves that this category is closed under smooth homotopies, contains algebras of the type $C^{\infty}(X)$ for a smooth manifold $X$, and for every admissible extension of $m$-algebras, whenever two of the terms in the extension are in $\mathcal{C}$, then so is the third.

Let us now consider a reductive $p$-adic group $G$. If $K$ is a compact open subgroup of $G$, then the Schwartz algebra $\mathcal{S}(G / / K)$ consists of all rapidly decaying K-bi-invariant functions on $G$ with complex values. The Schwartz algebra $\mathcal{S}(G)$ is the direct limit

$$
\mathcal{S}(\mathrm{G})=\lim _{\rightarrow} \mathcal{S}(\mathrm{G} / / \mathrm{K})
$$

equipped with the direct limit topology. We now define the Hecke algebra $\mathcal{H}(\mathrm{G} / / \mathrm{K})$ to be the subalgebra of the Schwartz algebra $\mathcal{S}(\mathrm{G} / / \mathrm{K})$ consisting of compactly supported functions. Again, the Hecke algebra $\mathcal{H}(\mathrm{G})$ is the direct limit of the algebras $\mathcal{H}(\mathrm{G} / / \mathrm{K})$. It should be noted that the Hecke algebra is considered in purely algebraic terms, without reference to topology.

Solleveld proves that the Schwartz algebras $\mathcal{S}(\mathrm{G} / / \mathrm{K})$ are objects of the category $\mathcal{C}$. The main result of [29] is the following.

Theorem 8.1. Let $\mathrm{G}$ be a reductive $\mathrm{p}$-adic group acting on an affine Bruhat-Tits building $\mathrm{X}$. There is a commutative diagram

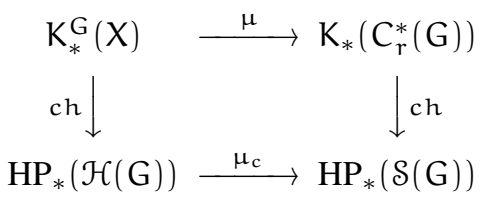


where the top horizontal arrow is the Baum-Connes assembly map, the vertical arrows are Chern characters, and the bottom horizontal arrows is induced by inclusion. Then $\mu_{c}$ is an isomorphism.

The proof of this result relies in part on Lafforgue's proof of the Baum-Connes conjecture for reductive $p$-adic groups [15], which shows that the map $\mu$ is an isomorphism. The vertical map on the left has been constructed in [1] where it was also proved that it is an isomorphism upon tensoring with $\mathbb{C}$. Given that the algebras $\mathcal{S}(\mathrm{G} / / \mathrm{K})$ belong to the category $\mathcal{C}$, the required isomorphism on the right (upon tensoring by $\mathcal{C}$ ) follows from the continuity of K-theory and the definition of the Schwartz algebra $\mathcal{S}(\mathrm{G})$. Finally, the map along the bottom edge is not affected by tensoring by $\mathcal{C}$, and so is an isomorphism.

\section{REFERENCES}

[1] P. Baum, N. Higson, R. J. Plymen, Representation theory of p-adic groups: a view from operator algebras, in: Proc. Sympos. Pure. Math. 68, American Mathematical Society, Providence RI, 2000, 111-149.

[2] J. Brodzki, R. Plymen, Entire cyclic cohomology of Schatten ideals. Homology, Homotopy Appl. 7 (2005), no. 3, 37-52.

[3] A. Connes, Noncommutative differential geometry. Inst. Hautes Études Sci. Publ. Math. 62 (1985), 257360 .

[4] A. Connes, H. Moscovici, Cyclic Cohomology, the Novikov Conjecture and Hyperbolic Groups, Topology 29 (1990), 345-388.

[5] J. Cuntz, A new look at KK -theory, K -Theory 1, 31-51 (1987).

[6] J. Cuntz, Excision in periodic cyclic theory for topological algebras. Cyclic cohomology and noncommutative geometry (Waterloo, ON, 1995), 43-53, Fields Inst. Commun. 17, Amer. Math. Soc., Providence, RI, 1997.

[7] J. Cuntz, G. Skandalis, B. Tsygan, Cyclic Homology in Noncommutative Geometry, Encyclopaedia of Mathematical Sciences 121, Springer Verlag, 2004.

[8] J. Cuntz, Bivariante K-Theorie für localkonvexe Algebren und der Chern-Connes-Charakter, Doc. Math. 2 (1997), 139-182.

[9] J. Cuntz, D. Quillen, Excision in bivariant periodic cyclic cohomology, Invent. math. 127 (1997), 67-98.

[10] J. Cuntz, D. Quillen, Cyclic homology and nonsingularity, Journal Amer. Math. Soc. 8 (1995) 373-442.

[11] J. Cuntz, Quillen's work on the foundations of cyclic cohomology; this issue.

[12] A. Grothendieck, Produits tensoriels topologiques et espaces nucléaires, AMS Memoir 16 (1966).

[13] G. Kasparov, G. Skandalis, Groupes boliques et conjecture de Novikov, C.R.A.S. 319 (1994), 815-820.

[14] C. Kassel, Caractère de Chern bivariant, K-Theory 3 (1989), 367-400.

[15] V. Lafforgue, K-théorie bivariante pour les algèbres de Banach et conjecture de Baum-Connes, Invent. Math. 149 (2002), 1-95.

[16] V. Mathai, D. Stevenson, On a generalized Connes-Hochschild-Kostant-Rosenberg theorem. Adv. Math. 200 (2006), no. 2, 303-335.

[17] V. Mathai, D. Stevenson, Entire cyclic homology of stable continuous trace algebras. Bull. Lond. Math. Soc. 39 (2007), no. 1, 71-75.

[18] R. Meyer, Cyclic cohomology theories and nilpotent extensions, Münster University thesis, Münster 1999, math.KT/9906205.

[19] R. Meyer, Local and Analytic Cyclic Homology. European Mathematical Society Publishing House, EMS Tracts in Mathematics 3, August 2007, 368 pages.

[20] R. Meyer, Excision in entire cyclic cohomology. J. Eur. Math. Soc. (JEMS) 3 (2001), no. 3, $269-286$.

[21] I. Mineyev, G. Yu, The Baum-Connes conjecture for hyperbolic groups, Invent. Math. 149 (2002), 97-122.

[22] V. Nistor, A bivariant Chern-Connes character. Ann. of Math. (2) 138 (1993), no. 3, 555-590.

[23] V. Nistor, On the Cuntz-Quillen boundary map. C. R. Math. Rep. Acad. Sci. Canada 16 (1994), no. 5, 203-208.

[24] V. Nistor, Higher index theorems and the boundary map in cyclic cohomology. Doc. Math. 2 (1997), 263295.

[25] M. Puschnigg, Excision in cyclic homology theories. Invent. Math. 143 (2001), no. 2, 249-323.

[26] M. Puschnigg, The Kadison-Kaplansky conjecture for word-hyperbolic groups. Invent. Math. 149 (2002), no. 1, 153-194.

[27] M. Puschnigg, Asymptotic cyclic cohomology. Lecture Notes in Mathematics 1642. Springer-Verlag, Berlin, 1996.

[28] D. Quillen, Cyclic cohomology and algebra extensions. K-Theory 3 (1989), no. 3, 205-246.

[29] M. Solleveld, Some Fréchet algebras for which the Chern character is an isomorphism. K-Theory 36 (2005), no. 3-4, 275-290. 
[30] C. Voigt. Equivariant local cyclic homology and the equivariant Chern-Connes character. Doc. Math. 12 (2007), 313-359 (electronic).

[31] C. Voigt. Equivariant periodic cyclic homology. J. Inst. Math. Jussieu 6 (4): 689-763, 2007.

[32] M. Wodzicki, Excision in cyclic homology and in rational algebraic K-theory, Ann. of Math. 129 (1989), 591-639.

Received: March 11, 2012

School of Mathematics, University of Southampton, Highfield, SOUthampton, SO17 1SH, ENGLAND

E-mail address: J.Brodzki@soton.ac.uk 